\title{
SCANDAL - A FACILITY FOR ELASTIC NEUTRON SCATTERING STUDIES IN THE 50-130 MeV RANGE
}

\author{
J. Blomgren*1, J. Klug ${ }^{1,5}$, P. Mermod ${ }^{1}$, L. Nilsson ${ }^{1}$, S. Pomp ${ }^{1}$, A. Öhrn ${ }^{1}$, Michael \\ Österlund $^{1}$, A.V. Prokofiev ${ }^{2}$, U. Tippawan ${ }^{1,3}$, J.F. Lecolley ${ }^{4}$, F.R. Lecolley ${ }^{4}$, N. \\ Marie-Noury $^{4}$, I. Sagrado-Garcia ${ }^{4}$, G. Ban ${ }^{4}$, G. Iltiss ${ }^{4}$, J.-M. Fontbonne ${ }^{4}$ \\ ${ }^{1}$ Department of Neutron Research, Uppsala University, Sweden \\ ${ }^{2}$ The Svedberg Laboratory, Uppsala University, Sweden \\ ${ }^{3}$ Fast Neutron Research Facility, Chiang Mai University, Thailand \\ ${ }^{4}$ LPC, ISMRA et Université de Caen, CNRS/IN2P3, France \\ ${ }^{5}$ Forschungszentrum Rossendorf, Dresden, Germany \\ E-mail: Jan.Blomgren@tsl.uu.se
}

\begin{abstract}
A facility for detection of scattered neutrons in the energy interval $50-130 \mathrm{MeV}$, SCANDAL (SCAttered Nucleon Detection AssembLy), is part of the standard detection system at the 20-180 $\mathrm{MeV}$ neutron beam facility of the The Svedberg Laboratory, Uppsala. It has primarily been used for studies of elastic neutron scattering, but it has been employed for $(n, p)$ and $(n, d)$ reaction experiments as well. Results of recent experiments are presented to illustrate the performance of the spectrometer.

Recently, the facility has been upgraded to perform also (n,Xn') experiments. For this purpose, a new converter, CLODIA, has been developed and installed. Preliminary results of the commissioning of CLODIA will be presented.
\end{abstract}

International Workshop on Fast Neutron Detectors and Applications

April, 3 - 6, 2006

University of Cape Town, South Africa

${ }^{*}$ Speaker. 


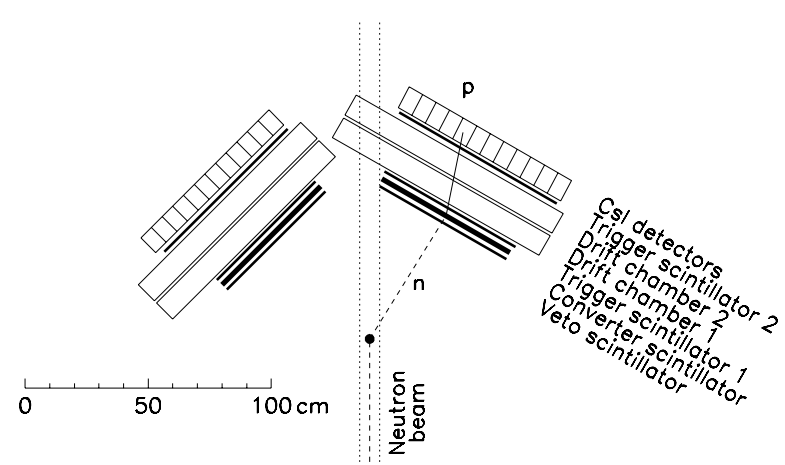

Figure 1: Overview of the SCANDAL setup.

\section{Introduction}

The development of new large-scale applications involving fast neutrons motivates nuclear data research. This applies to transmutation of spent nuclear fuel [1, 2, 3], neutron therapy of cancer tumours [4] and upsets in electronics [5, 6].

The SCANDAL (SCAttered Nucleon Detection AssembLy) setup [7] at The Svedberg Laboratory has been developed to meet some of these demands. The device has been designed for neutron elastic scattering experiments in the $50-130 \mathrm{MeV}$ range, but can be used also for charged-particle detection in the same energy interval. In the present paper, the focus is on the device. Thus, examples of the performance in various experiments are presented, while the physics results are presented elsewhere.

The neutron beam facility and the SCANDAL setup at The Svedberg Laboratory, Uppsala, Sweden, have recently been described in detail [7], and therefore only a brief description is given here. Neutrons are produced by the ${ }^{7} \operatorname{Li}(\mathrm{p}, \mathrm{n})$ reaction. The low-energy tail of the source-neutron spectrum is suppressed by time-of-flight techniques. After the target, the proton beam is bent into a well-shielded beam dump. A system of three collimators defines a $9 \mathrm{~cm}$ diameter neutron beam at the scattering target.

SCANDAL consists of two identical systems (see fig. 1). When used for neutron detection, they are normally placed to cover $10-50^{\circ}$ and $30-70^{\circ}$, respectively. The energy of the scattered neutrons is determined by measuring the energy of proton recoils from a plastic scintillator, and the angle is determined by tracking the recoil proton. Each arm consists of a $2 \mathrm{~mm}$ thick veto scintillator for fast charged-particle rejection, a $10 \mathrm{~mm}$ thick neutron-to-proton converter scintillator, a $2 \mathrm{~mm}$ thick plastic scintillator for triggering, two drift chambers for proton tracking, a $2 \mathrm{~mm}$ thick $\Delta E$ plastic scintillator that is also part of the trigger, and an array of CsI detectors for energy determination of recoil protons produced in the converter by $n p$ scattering. The trigger is provided by a coincidence of the two trigger scintillators, vetoed by the front scintillator. The total excitation energy resolution varies with CsI crystal, but is on average $3.7 \mathrm{MeV}$ (FWHM). The angular resolution is in the $1.0-1.3^{\circ}(\mathrm{rms})$ range.

When used for charged particle detection, the veto and converter scintillators are removed and a multi-target system is used to increase the count rate without deteriorating the energy resolution. 

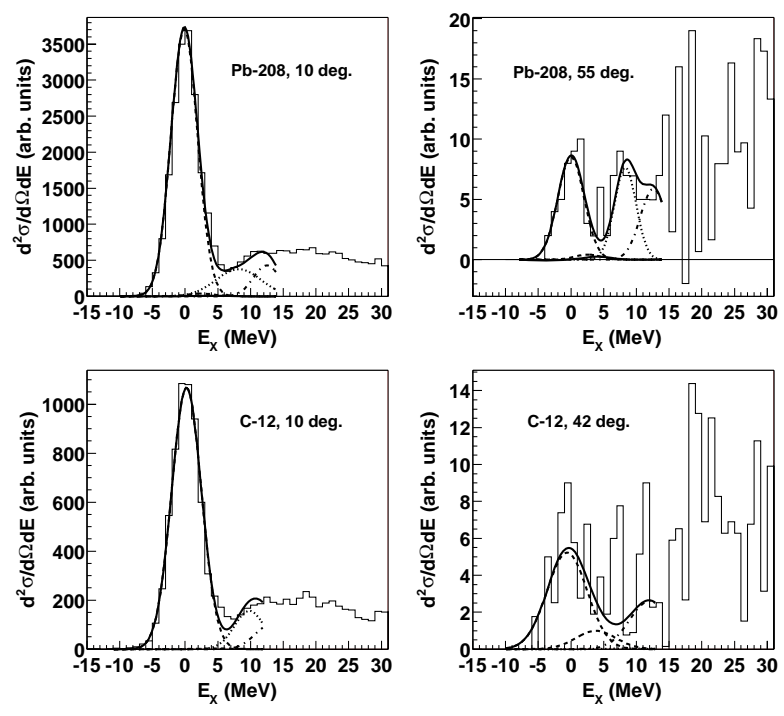

Figure 2: Excitation energy spectra for elastic neutron scattering from ${ }^{12} \mathrm{C}$ and ${ }^{208} \mathrm{~Pb}$ at $96 \mathrm{MeV}$ incident neutron energy, together with Gaussians representing known excited states.

\section{Elastic neutron scattering}

The main use of SCANDAL up to now has been elastic neutron scattering studies at $96 \mathrm{MeV}$. Examples of spectra on ${ }^{12} \mathrm{C}$ and ${ }^{208} \mathrm{~Pb}[8]$ are presented in fig. 2. As can be seen, the ground states are resolved. It is also evident that the cross section drops rapidly with angle. At the largest angles, around $70^{\circ}$, about one count per run week is obtained.

\section{3. (n,p) studies}

For transmutation applications, data on neutron-induced light-ion reactions are of importance, especially for assessment of materials damage in future accelerator-driven systems (ADS). Therefore, studies of (n,xlcp), where lcp denotes light charged particles, have been performed at $96 \mathrm{MeV}$ on iron, lead and uranium [9].

The MEDLEY facility [11] has been designed for such studies (see the contribution by Pomp et al.), but SCANDAL can be used to obtain additional information. The MEDLEY system operates in vacuum, which allows measurements of particle emission down to very low energies, and all light ions can be detected. SCANDAL operates in air, with the consequence that there is a $30 \mathrm{MeV}$ low-energy threshold for protons, and helium isotopes can hardly be detected at all. Nevertheless, the very large acceptance of SCANDAL makes it interesting for part of the emission spectrum. At backward angles, the high-energy proton emission is very weak, and in those regions SCANDAL can provide added value. An example is given in fig. 3.

\section{Neutron-deuteron scattering}

Neutron-deuteron scattering at intermediate energies has recently been identified as a good 

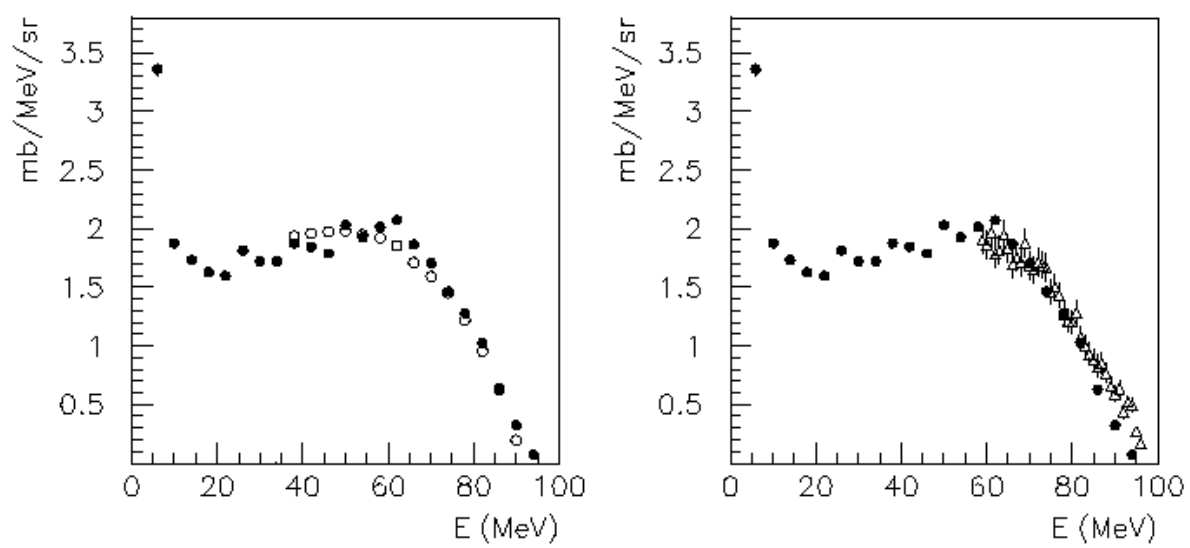

Figure 3: Left panel: $\mathrm{Fe}(\mathrm{n}, \mathrm{Xp})$ double-differential cross sections measured with the MEDLEY setup at $20^{\circ}$ (full circles), compared to the SCANDAL results (open circles). Right panel: The same data compared to those from Ref. [10] (open triangles).
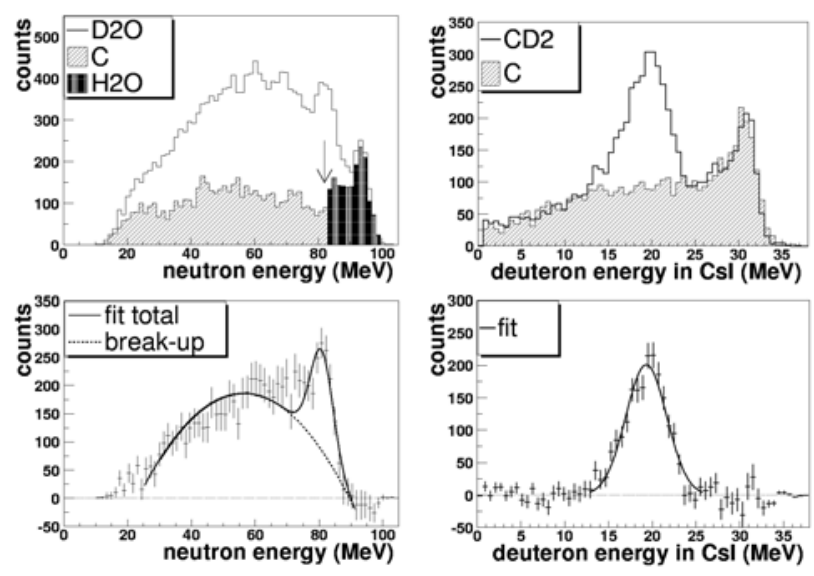

Figure 4: Typical energy spectra in $n d$ scattering experiments, showing neutrons detected at $30^{\circ}$ (left panels) and deuterons detected at $32^{\circ}$ (right panels). The bottom left panel shows the $n d$ spectrum after subtraction of the oxygen content in $\mathrm{D}_{2} \mathrm{O}$. The bottom right panel shows the nd elastic peak after subtraction of the carbon content in $\mathrm{CD}_{2}$. The error bars in the bottom panels are due to statistics.

case for studies of three-body effects in nuclei [12]. The full $n d$ angular distribution has been covered by two separate SCANDAL experiments. Forward angles have been studied by neutron detection, using heavy water targets for the signal, and light water targets for background. In addition, graphite targets were used for normalization. Backward angles were studied by deuteron detection, using $\mathrm{CD}_{2}$ discs for signal, pure graphite targets for background subtraction and $\mathrm{CH}_{2}$ targets for normalization.

Examples of spectra are presented in fig. 4. The final results show clear evidence of threenucleon force effects [13, 14]. Data obtained with the MEDLEY setup [15] are in agreement with the SCANDAL results. 


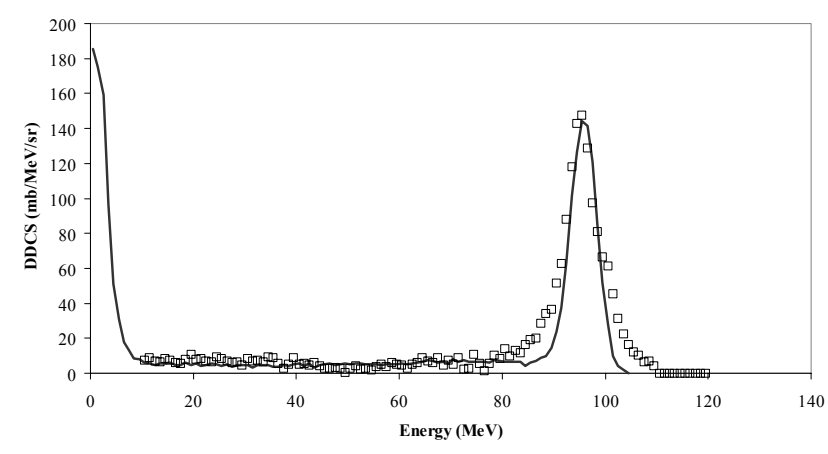

Figure 5: Preliminary results on $\mathrm{Pb}\left(\mathrm{n}, \mathrm{Xn}\right.$ ') at $95 \mathrm{MeV}$ energy and $15^{\circ}$, shown with an MCNPX prediction (black line).

\section{Inelastic neutron emission}

Like neutron-induced light ion emission, inelastic neutron emission is of large importance in the assessment of materials damage in future ADS applications. Only one high-quality neutroninduced data set exists above $30 \mathrm{MeV}$, a measurement at $65 \mathrm{MeV}$ of $\mathrm{Fe}, \mathrm{Sn}$ and $\mathrm{Pb}(\mathrm{n}, \mathrm{Xn}$ ') continuum cross sections at forward angles, however not beyond $30^{\circ}$ and with a $15 \mathrm{MeV}$ threshold [16].

The ( $\mathrm{n}, \mathrm{Xn}$ ') setup is composed of two separated devices, the DECOI/DEMON scintillators at low neutron energies $(10-50 \mathrm{MeV})$ and SCANDAL/CLODIA at high energies $(40-100 \mathrm{MeV})$, the latter being similar to the $65 \mathrm{MeV}$ system above. Low-energy neutrons interact with the hydrogen nuclei of the plastic scintillator DECOI, and scattered neutrons are then detected in the DEMON cell. Neutron identification is achieved via pulse-shape analysis [17], while the energy is derived from the time-of-flight of the scattered neutron between DECOI and DEMON. The upper threshold of $50 \mathrm{MeV}$ is a consequence of the path-flight $(100 \mathrm{~cm})$ while the lower one is mainly due to the low energy (about $50 \mathrm{keV}$ ) of the recoiling proton detected in DECOI.

High-energy neutrons are detected by $\mathrm{H}(\mathrm{n}, \mathrm{p})$ conversion in the CLODIA multi-layer converter, and the recoil protons are detected with SCANDAL. Neutron identification is achieved via rejection of incident charged particles using information from the veto scintillator and the first CLODIA drift chamber. The energy is derived from the tracking of the recoiling proton by several plastic scintillators and drift chambers, and the residual energy of those protons is measured in the SCANDAL CsI detectors. The low energy threshold of $40 \mathrm{MeV}$ is due to recoiling proton energy losses in the several planes of the set-up before they reach the CsIs.

Preliminary results are presented in fig. 5. Error bars are not yet available. Nevertheless, the good agreement in the energy region where the two devices overlap can be consider as a test of the whole procedure. Moreover, the elastic cross-section $\mathrm{Pb}(\mathrm{n}, \mathrm{n})$ derived from our preliminary results is in good agreement with a previous measurement perform at Uppsala [8]. In addition, MCNPX calculations using evaluated cross-sections calculated by GNASH for incident neutrons of $95 \mathrm{MeV}$ with a Gaussian distribution $(\Delta E=1.5 \mathrm{MeV})$ reproduce the preliminary results. 


\section{Acknowledgments}

This work was financially supported by the Swedish Nuclear Fuel and Waste Management Company, the Swedish Nuclear Power Inspectorate, Ringhals AB, Forsmarks Kraftgrupp AB, the Swedish Defense Research Agency, the Swedish Nuclear Safety and Training Centre, and the European Union.

\section{References}

[1] A. Koning, et al., J. Nucl. Sci. Tech., Suppl. 2 (2002) 1161.

[2] J. Blomgren, in Proceedings of Workshop on Nuclear Data for Science \& Technology: Accelerator Driven Waste Incineration, Trieste, Italy, Sept. 10-21, 2001, eds. M. Herman, N. Paver, A. Stanculescu, ICTP lecture notes 12 (2002) 327.

[3] J. Blomgren, Nuclear data for accelerator-driven systems - Experiments above $20 \mathrm{MeV}$, in Proceedings of EU enlargement workshop on Neutron Measurements and Evaluations for Applications, Bucharest, Romania, October 20-23, 2004.

[4] J. Blomgren and N. Olsson, Radiat. Prot. Dosim. 103(4) (2003) 293.

[5] J. Blomgren, B. Granbom, T. Granlund, N. Olsson, Mat. Res. Soc. Bull. 28 (2003) 121.

[6] J. Blomgren, Nuclear Data for Single-Event Effects, in Proceedings of EU enlargement workshop on Neutron Measurements and Evaluations for Applications, Budapest, Hungary, November 5-8, 2003. EUR Report 21100 EN, Luxembourg: Office for Official Publications of the European Communities, ISBN 92-894-6041-5, European Communities, 2004.

[7] J. Klug, et al., Nucl. Instr. Meth. A 489, 282 (2002).

[8] J. Klug, et al., Phys. Rev. C 68 (2003) 064605.

[9] V. Blideanu, et al., Phys. Rev. C 70 (2004) 014607.

[10] T. Rönnqvist, et al., Nucl. Phys. A563 (1993) 225.

[11] S. Dangtip, et al., Nucl. Instr. Meth. A452, 484 (2000).

[12] H. Witała, W. Glöckle, D. Hüber, J. Golak, H. Kamada, Phys. Rev. Lett. 81 (1998) 1183.

[13] P. Mermod, et al., Phys. Rev. C 72 (2005) 061002(R).

[14] P. Mermod, et al., submitted to Phys. Rev. C.

[15] P. Mermod, et al., Phys. Lett. B 597 (2004) 243.

[16] E.L. Hjort, et al., Phys. Rev. C 53 (1996) 237.

[17] I. Tilquin, et al., Nucl. Inst. Meth. Phys. Res. A 365 (1995) 446. 